significant Pfam hits, 4,520 sequences were in the cancer subclass. At present 59 Interpro entries exist in this subclass. These entries include breast cancer susceptibility proteins, retinoblastoma protein domains, p53 tumor antigen, Xeroderma pigmentosum proteins and the Burkitt's lymphoma receptor.

1. Apweiler R. et al. Nucl. Acids Res. 29, 37-40 (2001).

Yang, Ping

\section{Neutrophil elastase gene in lung cancer development: evidence from molecular genetics and clinical epidemiology}

Ping Yang, Ken Taniguchi, Claude Deschampes, Eric Bass, Rebecca Meyer \& Wanguo Liu

Mayo Foundation, Rochester, Minnesota 55901, USA

Neutrophil elastase (NE) is a powerful protease capable of degrading various protein components of the extracellular matrix, coagulation and component cascades. Local production of NE is involved in invasion and associated with a poor lung cancer prognosis. We propose that NE is important in lung cancer development, especially in the invasive phase. We tested this hypothesis using 344 cases and 299 controls. We detected single-nucleotide polymorphisms at the NE locus by denaturing high-performance liquid chromatography analysis, DNA sequencing and cloning of the NE gene promoter region. We performed transfection analysis, using relative luciferase activity on a human lung cancer cell line, to determine the regulatory role of the detected single-nucleotide polymorphisms in setting transcription levels of NE promoter. Two new single-nucleotide polymorphism markers, REP_A (T and G) and REP_B2 (G and A), were detected. There was no GG at REP_A and very little AA at REP_B2. TT and TG were contrasting allele types at REP_A, and GG and AA/AG were contrasting allele types at REP_B2. Measured by odds ratio, the TT type at REP_A or GG type at REP_B2 was associated with a 2.3 or 1.4 times higher risk of developing lung cancer than the TG and AA/AG types, respectively. When assessing the combined effects of the high-risk alleles, the odds ratio was 24.8. We demonstrated a 1.9-fold increase of relative luciferase activity in the T-G construct compared with the G-A construct, providing evidence that the TT-GG type correlates with a high NE level. Our findings indicate an important role for NE in lung cancer development.

\section{Monocytic leukemia zinc-finger protein MOZ and its related factor MORF are new histone acetyltransferases}

Nathalie Champagne, Nadine Pelletier \& Xiang-Jiao Yang

Molecular Oncology Group, McGill University Health Centre, Montreal, Quebec H3A 1G4, Canada

The monocytic leukemia zinc-finger protein $(\mathrm{MOZ})$ gene is rearranged in $\mathrm{t}(8$; 16)(p11; p13) and $\operatorname{inv}(8)(\mathrm{p} 11 \mathrm{q} 13)$ associated with acute myeloid leukemia. The other fusion partners involved are CBP and TIF2, both of which are known transcriptional coactivators with intrinsic histone acetyltransferase activity. We have cloned MORF, a new human protein related to $\mathrm{MOZ}$, and demonstrated that $\mathrm{MOZ}$ and MORF have intrinsic histone acetyltransferase activity. Moreover, like MORF, $\mathrm{MOZ}$ possesses a weak transcriptional repression domain at its $\mathrm{N}$-terminal part and a strong activation domain at its C-terminal part. These results indicate that $\mathrm{MOZ}$ and MORF are acetyltransferases involved in regulating transcription and thereby shed light on how aberrant MOZ proteins lead to leukemogenesis.
Yeung, Ka Yee

[69]

\section{Transcriptional analysis of Barrett's epithelium and normal gastrointestinal tissues}

Ka Yee Yeung ${ }^{1}$, Michael Barrett ${ }^{2}$, Jeff Delrow ${ }^{2}$, Patricia Blount $^{2}$, Brian Reid ${ }^{2} \&$ Peter Rabinovitch ${ }^{3}$

${ }^{1}$ Department of Computer Science and Engineering, University of Washington, Seattle, Washington, USA

${ }^{2}$ Fred Hutchinson Cancer Research Center, Seattle, Washington, USA

${ }^{3}$ Department of Pathology, University of Washington, Seattle, Washington, USA

Barrett's esophagus is a premalignant condition caused by chronic acid reflux in which the normal squamous epithelium of the esophagus is replaced by a metaplastic columnar epithelium. Of interest is the distinction between neoplastic Barrett's epithelium (BE) and surrounding normal tissues of the upper gastrointestinal tract. For example, although it arises in the esophagus, BE more closely resembles the epithelium of the duodenum at the histological level. We compared the transcriptional profile of $\mathrm{BE}$ to the profiles of normal upper gastrointestinal tissues, including gastric epithelium, squamous epithelium of the esophagus and duodenal epithelium. We collected endoscopic biopsies from each tissue from a series of patients during routine surveillance. Poly $(\mathrm{A})+\mathrm{RNA}$ was prepared from pooled samples (2-4 patients per pool) of BE (four pools), esophageal squamous epithelium (four pools), gastric epithelium (three pools) and duodenal epithelium (three pools) and used to interrogate Affymetrix HU6800 and FL6800 chips. We found no difference in the correlation coefficients between neoplastic BE and each of the normal tissues. In addition, we searched for tissue-specific patterns of gene expression in the normal tissues and the neoplastic BE. We compared the performance of several clustering algorithms. Our results suggest that the data set consists of approximately eight clusters, and the best performance was obtained using CAST and k-means. From this analysis, we applied CAST to identify eight clusters, among which some were specific for squamous epithelium (203 genes), duodenal epithelium (211 genes), gastric epithelium (105 genes) and BE (36 genes).

Yuan, Bo

[70]

\section{Physical mapping and functional annotation of 60,000 human genes}

Degen Zhuo ${ }^{1}$, Wei Zhao ${ }^{1}$, Hee-Yung Yang ${ }^{2}$, Jian-Ping Wang ${ }^{1}$, Russell Sears ${ }^{1}$, Do-Hun Kwon ${ }^{1}$, David Gordon ${ }^{1}$, Solomon Gibbs ${ }^{1}$, Dai Dean ${ }^{2}$, Troy Baer ${ }^{3}$, Don Stredney ${ }^{3}$, Al Stutz ${ }^{3}$, Ralf Krahe ${ }^{1}$, Fred Wright ${ }^{1} \&$ Bo Yuan $^{1}$

\footnotetext{
${ }^{1}$ Bioinformatics Group, Human Cancer Genetics Program, Ohio State University, Columbus, Ohio, USA

${ }^{2}$ Labbook.com, Columbus, Ohio, USA

${ }^{3}$ Ohio Supercomputer Center, Columbus, Ohio, USA
}

The recent release of the first draft of the human genome provides an unprecedented opportunity to integrate all human genes and their functions within a complete positional context. However, at least four significant technical hurdles remain: to create a complete and nonredundant human transcript index, to assemble the still-fragmented human genome draft, to place the individual transcript indices accurately on the human genome and to annotate all human genes functionally. We report the extension of the UniGene database through the assembly of its sequence clusters into nonredundant sequence contigs. The resulting consensus was aligned to the draft genome. We determined a unique location for each transcript within the human genome by the integration of the restriction fin- 